\title{
Progress in X-ray Mapping in Electron Microscopes Toward Single-Atom Analysis
}

\author{
M. Watanabe ${ }^{1}$
}

1. Dept of Materials Science and Engineering, Lehigh University, Bethlehem. PA, USA.

Since the first development and application by Castaing, X-ray analysis has been performed in scanning electron microscopes (SEMs)/electron probe microanalysers (EPMAs) mainly for bulk samples [1] and in analytical electron microscopes (AEMs) for electron transparent thin-film specimens [2]. The X-ray signals excited by primary electrons have also been used to compose elemental and/or compositional images, called as X-ray maps, in which compositional fluctuations in any lateral direction can be visualized. The X-ray mapping technique was developed by Peter Duncumb first in a converted transmission electron microscope (TEM) [3], and immediately applied to maps light elements (Be, C, and O) with a peak separation scheme by Ray Dolby [4]. These initial demonstrations of X-ray mapping in the SEM were conducted under supervision of Ellis Cosslett at the Cavendish Laboratory in UK. $\mathrm{X}$-ray mapping in SEMs/EPMAs is obviously one of the essential characterization tools used widely to extract quantitative information from various materials.

Spatial resolution of X-ray analysis has been dramatically improved by using AEMs in combination with thin specimens. This improved spatial resolution in AEMs offsets the analytical sensitivities of $\mathrm{X}$-ray analysis due to the limited analysed-volume and to restricted interface designs between an AEM column and X-ray detectors. Therefore, X-ray mapping has not generally been successful in AEMs for many years. However, these limits of the poor X-ray generation and poor X-ray collection were overcome in some degree by employing a high brightness electron source and a modified geometry of X-ray detectors, which were incorporated in some instruments in late '90s, e.g. the HB 603 AEM at Lehigh. Using this instrument, X-ray maps were able to be obtained with improved spatial resolution and analytical sensitivity [5].

Based on the results obtained from the HB 603 and other instruments, a prediction of the next generation X-ray microanalysis by AEMs was plotted as the shadowed area, together with a prediction of next generation SEMs/EPMAs estimated by Newbury et al. [6] in Fig. 1, which is modified from previously published plots [5]. Further progresses have been made since these predictions. In current SEMs/EPMAs, operations at much lower accelerating voltages (even below $1 \mathrm{kV}$ ) are possible, which improves spatial resolution of X-ray analysis, down to a few nm levels, if soft X-ray lines are used. For AEMs, the spatial resolution of X-ray analysis is more significantly improved, down to $1 \AA$ level due to advances of the latest aberration correction technologies. Additionally, development of large solid angle silicon-drift X-ray detectors (SDDs) and their multiple arrangements also improve poor signal collection efficiency, and hence the analytical sensitivity. By using the aberration-corrected AEM with larger solid-angle SDDs, single-atom analysis has been proven by X-ray analysis [7], which requires atomic-level spatial resolution in combination with a sensitivity of single atom detection. Figure 2 compares the first X-ray map by Duncumb [3] and an atomic-resolution X-ray map measured by the aberration-corrected JEM-ARM200CF AEM at Lehigh. Over 50 years progress, X-ray analysis has reached the ultimate detection limit of physical techniques for microanalysis as predicted by Cliff and Kenway [8], and as addressed by Wittry [9] [10]. 


\section{References:}

[1] J.I. Goldstein, et al., Scanning Electron Microscopy and X-ray Microanalysis, $3^{\text {rd }}$ Ed. Plenum Press, New York, (2003).

[2] D.B. Williams \& C.B. Carter. Transmission Electron Microscopy, $2^{\text {nd }}$ ed. Springer, NY (2009).

[3] V.E. Cosslett \& P. Duncumb, Nature 177 (1956), p. 1172.

[4] R.M.Dolby, Proc. ICXOM3 1962, Academic Press, 483 (1963)

[5] D.B. Williams et al, J. Electron Microscopy 51S, S113 (2002).

[6] D.E. Newbury et al, Ultramicroscopy 78 (1999), p. 73.

[7] T. Lovejoy et al, Appl.Phys. Lett. 100 (2012), p. 154101.

[8] G. Cliff \& P.B. Kenway, Proc. $47^{\text {th }}$ Annual Meeting EMSA (1989) p. 200

[9] D.B. Wittry, Electron Microscopy 3 (1980), p. 14.

[10] The author wishes to acknowledge financial support from the NSF through grants DMR-0804528 and DMR-1040229, and would like to thank to Dr. Peter Duncumb for providing useful information and reviewing this paper.

Fig. 1

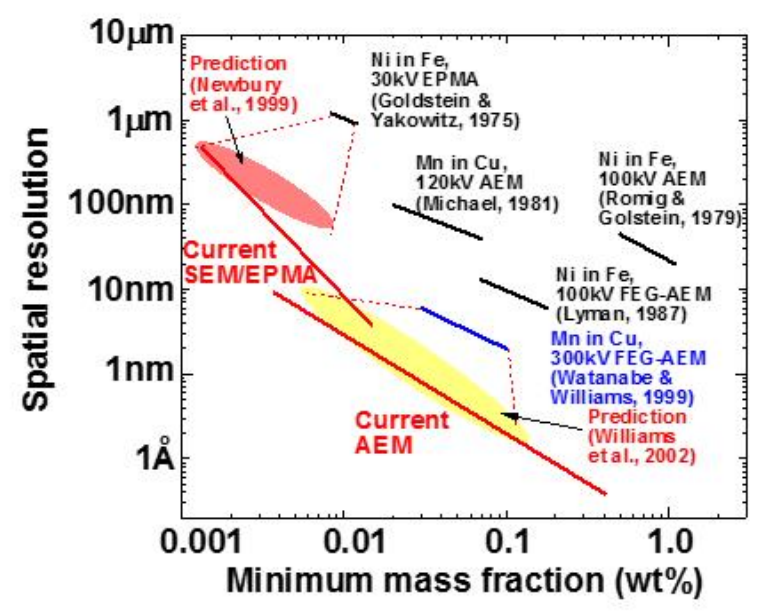

Fig. 2

(a)

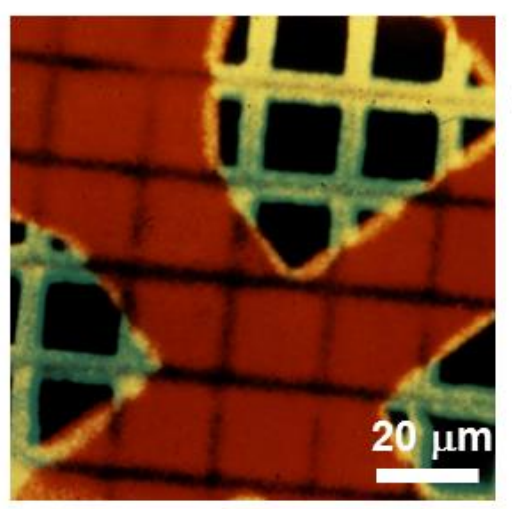

(b)

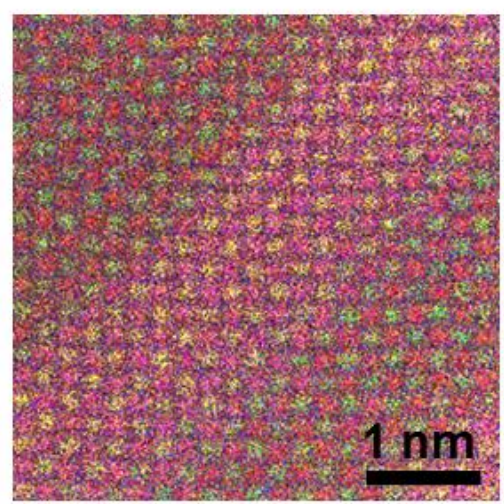

Figure 1. A summary of the relationship between the spatial resolution and the analytical sensitivity in term of the minimum mass fraction for the X-ray analysis in several electron-probe instruments, modified from the original plot [4].

Figure 2. Comparison of X-ray maps: (a) the first $\mathrm{X}$-ray map of $\mathrm{Cu}$ and $\mathrm{Ag}$ grids (Cu: red and $\mathrm{Ag}$ : yellow) by Duncumb [3] and (b) an atomic-resolution X-ray map of a $\mathrm{SrTiO}_{3} / \mathrm{LaMnO}_{3}$ multilayer obtained by the aberration-corrected JEM-ARM200CF AEM at Lehigh (Sr: red, Ti: green, O: blue, La: magenta and Mn: yellow). 